\title{
Microbes on the Mobile Phones of Healthcare Workers in Palestine: Identification, Characterization, and Comparison
}

\author{
Mohammad Qadi (D), ${ }^{1}$ Rasha Khayyat $\left(D,{ }^{1}\right.$ Mohammed A. AlHajhamad $\left(D^{2},{ }^{2}\right.$ Yazan I. Naji (iD), \\ Beesan Maraqa $\mathbb{D}^{3},{ }^{3}$ Kais Abuzaitoun $\mathbb{D}^{2},{ }^{2}$ Ahmed Mousa $\mathbb{D}^{1},{ }^{1}$ and Maysa Daqqa $\mathbb{D}^{1}$ \\ ${ }^{1}$ Department of Biomedical Sciences, Faculty of Medicine and Health Sciences, An-Najah National University, Nablus, \\ P.O. Box 7, State of Palestine \\ ${ }^{2}$ Department of Medicine, Faculty of Medicine and Health Sciences, An-Najah National University, Nablus, P.O. Box 7, \\ State of Palestine \\ ${ }^{3}$ Primary Health Directorate, Palestinian Ministry of Health, Nablus, State of Palestine \\ Correspondence should be addressed to Mohammad Qadi; m.qadi@najah.edu
}

Received 6 September 2020; Revised 3 February 2021; Accepted 18 February 2021; Published 26 February 2021

Academic Editor: Gabriele Messina

Copyright (c) 2021 Mohammad Qadi et al. This is an open access article distributed under the Creative Commons Attribution License, which permits unrestricted use, distribution, and reproduction in any medium, provided the original work is properly cited.

\begin{abstract}
Background. Healthcare workers (HCWs) may be using their mobile phones (MPs) to carry microbes that cause hospital-acquired and community infections in general. With antibiotic resistance problem emergence, these infections can be challenging to eradicate. Hence, this study aimed to determine the microbial contamination of HCW MPs and identify and classify bacterial isolates in Palestine. Methods. This was a 7-month comparative cross-sectional analysis of $200 \mathrm{HCW}$ MPs from 2 hospitals and 100 MPs from university students (non-HCWs). Data collection was done using a self-administrated questionnaire, and a swab sample from both HCW and non-HCW MPs was obtained and transferred to An-Najah National University (NNU) microbiology lab for bacterial identification and antibiotic susceptibility. Data were analyzed using Social Sciences Statistical Package (SPSS) version 22.0. Result. Among HCWs, the microbial contamination was 87.5\%. Coagulase-negative staphylococci (CoNS; 67.3\%), methicillin-sensitive Staphylococcus aureus (MSSA; 17.5\%), Gram-positive bacilli (4.1\%), methicillin-resistant Staphylococcus aureus (MRSA; 1.6\%), and Gram-negative species (1.6\%) were the most predominant bacterial isolates. More than half of staphylococci isolates were resistant to penicillin and erythromycin. Male gender, using a mobile phone in the bathroom, and entry to the operating theatre were associated with mobile phone contamination and increased resistance against specific antibiotics. Among non-HCWs, the contamination was $86 \%$. The most predominant bacterial isolates were CoNS, MSSA, and Gram-positive bacilli, with a contamination of $66.8 \%, 28.5 \%$, and $2.6 \%$, respectively. No MRSA or Gram-negative species were detected in this group. Antibiotic resistance percentage of staphylococci was nearly half of that yielded in the HCW group against each antibiotic. Conclusion. Significant numbers of bacteria have been isolated from HCW MPs. Working in a hospital environment frequently raises the probability of presence of antibiotic-resistant bacteria on a MP. Therefore, infection control teams should discuss methods to prevent the transmission of drug-resistant pathogens from HCW MPs.
\end{abstract}

\section{Introduction}

A nosocomial infection (NI) is a severe global problem and contributes significantly to patient morbidity and mortality [1]. Mobile phones (MPs) are essential in everyday life and are carried everywhere and at all times [2]. Moreover, they are always in close contact with the hands and body skin [3]. MPs are also becoming an essential tool in medical practice.
First, they are primarily used in communication among healthcare workers (HCWs), making healthcare work more efficient [4]. Secondly, they provide medical information access by asking for consultation and reaching medical references [2]. However, MPs are rarely disinfected and are often touched by HCWs before, during, and after examining their patients without handwashing [3]. Many studies reported the colonization of potentially pathogenic organisms 
on various objects as hands, MPs, personal digital assistants, computer keyboards, and pagers. These objects have been suggested as possible vectors for the transmission of nosocomial pathogens from HCWs to patients as well as severe acute respiratory syndrome coronavirus 2 (SARS-CoV-2) [5-8]. Contaminated MPs are potential vectors for spreading microbes in both hospitals and communities, making them an exogenous source of nosocomial infections [9-12].

The NI affects vast numbers of patients globally, significantly raising mortality rates and financial losses. Figures show that about $15 \%$ of all hospitalized patients suffer from these infections. In high-income countries, the incidence is high enough, between $3.5 \%$ and $12 \%$, while in middle and low-income countries (LMICs), such as Palestine, it varies from $5.7 \%$ to $19.1 \%$ [13]. NIs caused by multidrug resistant bacteria are a growing problem in many healthcare institutions [13]. Instruments, hands, MPs, and other inanimate hospital objects used by the HCW may serve as vehicles and reservoirs for the NIs. However, the incidence of such infections can be reduced by maintaining proper hygiene between the HCW and the hospital environment $[1,14,15]$.

MPs have become multipurpose nonmedical devices used in the healthcare facility and the community. They have increasingly become an essential means of communication in the community and healthcare facilities to collect epidemiological data and monitor chronic diseases $[15,16]$. MPs are used without restriction in healthcare facilities regardless of their microbial load. Attempting to avoid NIs, it is worth studying and identifying pathogens on MPs to improve the quality of healthcare. MPs' potential to become a nosocomial infection source has been studied before $[6,17,18]$. However, to the best of our knowledge, no study or published data have been done in Palestine. This research aims to analyze and compare the degree of contamination of HCW members with non-HCW members. In addition, it aims to examining the prevalence of antibiotic-resistant bacteria on MPs' and correlate it to handling habits.

\section{Materials and Methods}

2.1. Study Design and Setting. This is a comparative crosssectional study of the frequency of bacterial contamination of HCW and non-HCW participants between September 2018 and March 2019. This study was performed in Nablus, situated in the northern part of the West Bank, Palestine.

2.2. Study Population. In this study, the participants' MPs were sampled without any prior intimation and divided into two groups: the HCW group and the non-HCW group. We recruited HCW participants from two separate hospitals in Nablus, West Bank, Palestine: Rafidia Surgical Hospital as a governmental hospital and An-Najah National University Hospital (NNUH) as a nongovernmental hospital. We considered the governmental hospital as hospital A and the nongovernmental hospital as hospital B. On the other hand, the non-HCW group comprises engineering and IT students at An-Najah National University (ANNU) since their field depends on using MPs more frequently and they have no interaction with the healthcare system during their study.

2.2.1. Inclusion Criteria. HCWs were physicians, residents in training, nurses, and any other person who has a hospital position as lab technicians and radiology staff. Consenting participants were asked to enroll their phones, choosing the most frequently used device, in case of multiple eligible devices.

2.2.2. Exclusion Criteria. We excluded personnel who do not possess or use MPs at the time of analysis and those who cleaned their phones once they heard about our study, and those whose MPs were contaminated by our hands during sample collection were also excluded.

2.3. Sample Size. By considering the following statistical assumptions, the sample size for this analysis uses a double population proportion formula: $95 \%$ confidence level, the prevalence of contamination is $50 \%$ and $5 \%$ error margin, and a power of $80 \%$. Rosoft sample size calculator was used. The total population of healthcare staff in both hospitals was 400 , and half of the HCW's contamination was anticipated in non-HCW's. As a result, samples of 200 from MPs of HCW and 100 from MPs of non-HCW were enrolled.

2.3.1. Questionnaire. Consent participants were asked to complete a questionnaire before taking a swab sample from their MPs. The questionnaire addressed participants' usage habits by several domains: their daily use and a special section asking about using their MPs in hospital and medical practice. Only the HCW has filled this particular section. The questionnaire was written in the local language, Arabic. It included questions about gender, age, and average time spent daily on MPs. Other questions assessed the frequency of handwashing per day, frequency of MP cleaning per month, last time of MP cleaning, and MP cleaning method. Besides, questions set smoking status, using MP in the bathroom, and broken quality of the screen or the screen protector.

A special section asked HCWs about their position at the hospital, availability of antiseptic solutions in the wards, using mobile on the entrance to operating rooms, using the MPs in the operating theatre, and using MPs next to the patient.

To ensure its validity and reliability, the questionnaire was pretested before the finalization. The questionnaire was checked by experts from An-Najah National University Faculty who suggested adding several questions.

A pilot study was performed on 20 samples for both training purposes on the sampling process and to check the questionnaire's simplicity and readability and the time it takes to be filled.

2.3.2. Sample Collection. A sterile cotton swab with a transport medium was used to take a swab from the touchscreen, mouthpiece, earpiece, and buttons since these 
areas are the most frequent spots in contact with the fingers, as done in other studies [3]. After swabbing, samples were transferred immediately and adequately within 2 hours to the NNUH microbiology lab for culturing on suitable media (blood agar and MacConkey). Then, the inoculated media were incubated at $37^{\circ} \mathrm{C}$ for 48 hours.

2.3.3. Identification of Microorganisms. After incubation time was over, colonies that grew on plated media were subcultured to obtain a pure culture from each isolated type of colony. Then, isolated microorganisms were identified using different microbiological identification approaches, including morphological and biochemical features.

Briefly, all isolates were subjected to Gram stain, and based on the result, identification was performed as follows:

(i) A spore staining procedure was applied for such isolates as Gram-positive bacilli. This was for allowing differentiation into spore-forming and non-spore-forming Gram-positive bacilli.

(ii) For Gram-positive cocci, isolates were subjected to catalase test, and those with positive reaction were cultured on mannitol salt agar to classify them into $S$. aureus and CoNS. At the same time, catalasenegative isolates were considered as other Grampositive cocci.

(iii) A glucose fermentation test was applied for Gramnegative bacilli to group them into glucose fermenters and glucose nonfermenters.

Finally, based on the mentioned adapted identification scheme, isolated microbes were grouped for data analysis.

2.3.4. Antibiotic Sensitivity Test. This test was performed by the Kirby-Bauer disc diffusion method on Mueller-Hinton agar according to Clinical Laboratory Standards Institute 2018 antibiotic disc susceptibility testing guidelines. Antibiotic sensitivity profile was done only for Staphylococcus species. Antibiotics used for this group of bacteria were as follows: penicillin (P), clindamycin (DA), cefoxitin (FOX), erythromycin (E), and trimethoprim-sulfamethoxazole (SXT). $S$. aureus isolates resistant to FOX were considered as methicillin-resistant $S$. aureus (MRSA) and were, afterward, tested with 30-microgram vancomycin disc on Mueller-Hinton agar to test for the existence of vancomycin-resistant $S$. aureus.

2.4. Data Analysis. The Statistical Package of Social Sciences (SPSS) v. 22.0 software was used to analyze the obtained data. Using tables and figures when needed, a descriptive analysis of sample results was presented. Using the chisquare test, the association between research variables was evaluated. The agreed significance level has been set below $0.05(p<0.05)$.

2.5. Ethical Consideration. Approval was obtained from the IRB (Institutional Review Board) at NNU before initiating this research. The privacy and confidentiality of the participants were ensured. After participants read the research overview, informed consent was obtained orally, and the participants were informed that their involvement in the study was voluntary.

\section{Results}

In this study, 200 samples of HCWs and 100 samples of nonHCWs were obtained. Of those who completed the questionnaire, more than half of the participants were males, as shown in Table 1.

MP-related habits and comparisons of the questionnaire data of HCWs with non-HCWs are presented in Table 1. Daily time spent on the phone showed considerable variation between the two groups; spending more than two hours on MPs was more common among HCWs. On the other hand, the handwashing level of HCWs was substantially higher than that of non-HCWs. The use of alcohol in parliamentary cleaning was considerably more common among HCWs.

Samples of HCWs were collected from specialists $(10.6 \%)$, nursing staff $(44 \%)$, medical residents $(28.2 \%)$, and other healthcare professionals such as technicians working in various departments: laboratory, radiology department, optometry, operating theatre, and general wards (13.6\%). Relevant scenarios in which the HCW could use their MPs and contribute NIs were described in the questionnaire, as shown in Table 2. HCWs demonstrated that most of them insisted on the availability of hand sanitizer at all times in the facility they serve. When entering the operating room, doctors (specialists and residents) were more likely to use their MPs, while residents were much more likely to use their mobile phones alongside patients.

3.1. Prevalence and Type of Bacterial Isolates. A total of 300 mobile phone samples were examined for the presence of bacterial contamination. Bacterial contamination was found in swabs taken from 175 HCW MPs (87.5\%) and 86 nonHCW MPs (86\%). No significant difference was noticed in the study's microbial contamination between the HCW and control group non-HCW. The total number of bacterial isolates was 628 from both groups.

From the $200 \mathrm{HCW}$ mobile phones, 435 bacterial isolates were obtained and characterized. Figure 1 illustrates the different types of bacteria isolated and their distribution. Four hundred twenty-eight bacterial isolates were found to be Gram-positive. Among which, 293 bacteria were CoNS (67.3\%), 76 were methicillin-sensitive Staphylococcus aureus (MSSA) (17.5\%), 13 were non-spore-forming Gram-positive bacilli (3\%), 5 were spore-forming Gram-positive bacilli $(1.2 \%)$, and 34 were other Gram-positive cocci (7.9\%). Seven bacterial isolates were found to be Gram-negative bacteria $(1.6 \%)$, among which 3 were glucose fermenters $(0.7 \%)$ and 4 were non-glucose fermenters $(0.9 \%)$.

Table 3 illustrates the significant differences between hospitals A and B concerning isolated bacteria. No significant differences were noted between the two hospitals. In hospital A, MRSA, Gram-negative species, and Gram-positive bacilli percentages were higher. 
TABLE 1: Background variables of the participants.

\begin{tabular}{|c|c|c|c|}
\hline \multirow[b]{2}{*}{ Variable } & \multicolumn{2}{|c|}{ Participant characteristic } & \multirow[b]{2}{*}{$p$ value } \\
\hline & $\begin{array}{c}\text { Non-healthcare worker }(n=98) \\
\text { Frequency }(\%)\end{array}$ & $\begin{array}{c}\text { Healthcare workers }(n=195) \\
\text { Frequency }(\%)\end{array}$ & \\
\hline \multicolumn{4}{|l|}{ Gender } \\
\hline Male & $52(53.1 \%)$ & $111(56.9 \%)$ & \multirow{3}{*}{0.63} \\
\hline Female & $46(46.9 \%)$ & $84(43.1 \%)$ & \\
\hline Missing & $0(0 \%)$ & $0(0 \%)$ & \\
\hline \multicolumn{4}{|c|}{ Time spent using the phone on average } \\
\hline$<60$ minutes & $12(12.2 \%)$ & $67(34.4 \%)$ & \multirow{4}{*}{$<0.001$} \\
\hline $60-120$ minutes & $18(18.4 \%)$ & $41(21.0 \%)$ & \\
\hline$>120$ minutes & $68(69.4 \%)$ & $86(44.1 \%)$ & \\
\hline Missing & $0(0 \%)$ & $1(0.5 \%)$ & \\
\hline \multicolumn{4}{|c|}{ Number of times of handwashing daily } \\
\hline$\leq$ Four times & $32(32.7 \%)$ & $29(14.9 \%)$ & \multirow{4}{*}{$<0.001$} \\
\hline $5-7$ times & $33(33.7 \%)$ & $42(21.5 \%)$ & \\
\hline$>7$ times & $32(32.6 \%)$ & $123(63.1 \%)$ & \\
\hline Missing & $1(1 \%)$ & $1(0.5 \%)$ & \\
\hline \multicolumn{4}{|c|}{ Frequency of phone cleaning } \\
\hline Daily & $09(9.2 \%)$ & $48(24.6 \%)$ & \multirow{5}{*}{0.008} \\
\hline Weekly & $38(38.8 \%)$ & $76(39 \%)$ & \\
\hline Monthly & $16(16.3 \%)$ & $21(10.8 \%)$ & \\
\hline Rarely & $34(34.7 \%)$ & $48(24.6 \%)$ & \\
\hline Missing & $1(1 \%)$ & $2(1 \%)$ & \\
\hline \multicolumn{4}{|c|}{ Last time phone cleaning } \\
\hline Last three days & $37(37.8 \%)$ & $77(39.4 \%)$ & \multirow{5}{*}{0.97} \\
\hline $3-6$ days ago & $21(21.4 \%)$ & $39(20.0 \%)$ & \\
\hline A week or more & $18(18.4 \%)$ & $33(17.0 \%)$ & \\
\hline A month or more & $22(22.4 \%)$ & $45(23.1 \%)$ & \\
\hline Missing & $0(0 \%)$ & $1(0.5 \%)$ & \\
\hline \multicolumn{4}{|c|}{ Method of cleaning the phone } \\
\hline Alcohol & $11(11.2 \%)$ & $112(57.4 \%)$ & \multirow{5}{*}{$<0.001$} \\
\hline Water & $15(15.3 \%)$ & $6(3.1 \%)$ & \\
\hline Wet wipes & $52(53.1 \%)$ & $48(24.6 \%)$ & \\
\hline Paper wipes & $20(20.4 \%)$ & $25(12.8 \%)$ & \\
\hline Missing & $0(0 \%)$ & $4(2.10 \%)$ & \\
\hline \multicolumn{4}{|l|}{ Smoking } \\
\hline Yes & $11(11.2 \%)$ & $46(23.6 \%)$ & \multirow{3}{*}{0.017} \\
\hline No & $86(87.8 \%)$ & $149(76.4 \%)$ & \\
\hline Missing & $1(1 \%)$ & $0(0 \%)$ & \\
\hline \multicolumn{4}{|c|}{ Use of phone in the bathroom } \\
\hline Yes & $45(45.9 \%)$ & $78(40.0 \%)$ & \multirow{3}{*}{0.36} \\
\hline No & $50(51.1 \%)$ & $112(57.5 \%)$ & \\
\hline Missing & $3(3 \%)$ & $5(2.5 \%)$ & \\
\hline \multicolumn{4}{|c|}{ Breaks in the screen or screen protector } \\
\hline Yes & $38(38.8 \%)$ & $79(40.5 \%)$ & \multirow{3}{*}{0.58} \\
\hline No & $57(58.1 \%)$ & $110(56.4 \%)$ & \\
\hline Missing & $3(3.1 \%)$ & $6(3.1 \%)$ & \\
\hline
\end{tabular}

From the 100 non-HCW MPs, 193 bacteria were isolated. Interestingly, those isolates were found to be only Grampositive bacteria. Among which, 129 were CoNS (66.8\%), 55 were MSSA (28.5\%), 5 were non-spore-forming Gram-positive bacilli (2.6\%), and 4 were other Gram-positive cocci $(2.1 \%)$. There were no MRSA, no spore-forming Gram-positive bacilli, or Gram-negative bacteria isolated. The distribution of bacteria from the non-HCW is shown in Figure 2.

3.2. Antibiotic Susceptibility Pattern. Antibiotic susceptibility pattern of Staphylococci isolated from HCW and nonHCW MPs is shown in Table 4. Regarding the susceptibility of $S$. aureus in HCW MPs, cefoxitin was the most effective antibiotic (susceptibility $=82.1 \%$ ). Other antibiotics came next: clindamycin $(S=67.2 \%)$, trimethoprim-sulfamethoxazole $(S=64.2 \%)$, erythromycin $(S=23.9 \%)$, and penicillin, which was the least influential $(S=14.9 \%)$. For CoNS, cefoxitin was also the most effective antibiotic $(S=91.3 \%)$. It was followed by trimethoprim-sulfamethoxazole $(S=72.5 \%), \quad$ clindamycin $\quad(S=64.9 \%), \quad$ erythromycin $(S=23.5 \%)$, and penicillin, which was the least influential $(S=19.5 \%)$.

Furthermore, for $S$. aureus in non-HCWs, cefoxitin was the most effective antibiotic $(S=96.4 \%)$. For trimethoprimsulfamethoxazole, it was $S=85.7 \%$, clindamycin showed 
TABLE 2: The use of MPs by HCWs in unique characteristic scenarios.

\begin{tabular}{|c|c|c|c|c|c|}
\hline \multirow[t]{2}{*}{ Scenario } & \multicolumn{5}{|c|}{$\mathrm{HCW}$} \\
\hline & $\begin{array}{c}\text { Specialist } \\
(N=21) \\
n(\%)\end{array}$ & $\begin{array}{c}\text { Resident } \\
(N=56) \\
n(\%)\end{array}$ & $\begin{array}{c}\text { Nurse } \\
(N=86) \\
n(\%)\end{array}$ & $\begin{array}{l}\text { Other HCWs } \\
\begin{array}{c}(N=27) \\
n(\%)\end{array}\end{array}$ & $p$ value* \\
\hline \multicolumn{6}{|c|}{$\begin{array}{l}\text { The constant presence of hand sanitizer in the medical } \\
\text { facility** }\end{array}$} \\
\hline Yes & $19(90.3)$ & 49 (87.5) & $84(96.6 \%)$ & $25(92.6 \%)$ & \multirow{2}{*}{0.126} \\
\hline No & $2(9.5)$ & $7(12.5)$ & $2(2.40 \%)$ & $2(7.4 \%)$ & \\
\hline \multicolumn{6}{|c|}{ Using mobile on entry to operating theatre ${ }^{* *}$} \\
\hline Yes & $12(57.1)$ & $38(67.9)$ & $33(38.4)$ & $5(18.5)$ & \multirow{2}{*}{$<0.001$} \\
\hline No & $9(42.9)$ & $18(32.1)$ & $53(61.6)$ & $22(81.5)$ & \\
\hline \multicolumn{6}{|c|}{ Usage of mobile phone in the operating theatre** } \\
\hline Yes & $7(33.3)$ & $23(41.1)$ & $10(11.8)$ & $3(11.1)$ & \multirow{2}{*}{$<0.001$} \\
\hline No & $12(57.1)$ & $31(55.4)$ & $72(83.5)$ & $20(74.1)$ & \\
\hline \multicolumn{6}{|c|}{ Usage of the mobile phone next to patients** } \\
\hline Yes & $7(33.3)$ & $34(60.7)$ & $34(39.5)$ & $10(37.0)$ & \multirow{2}{*}{0.026} \\
\hline No & $14(66.7)$ & $22(39.3)$ & $52(60.5)$ & $16(59.2)$ & \\
\hline
\end{tabular}

${ }^{*}$ Chi-square test, ${ }^{* *}$ missing $=10$.

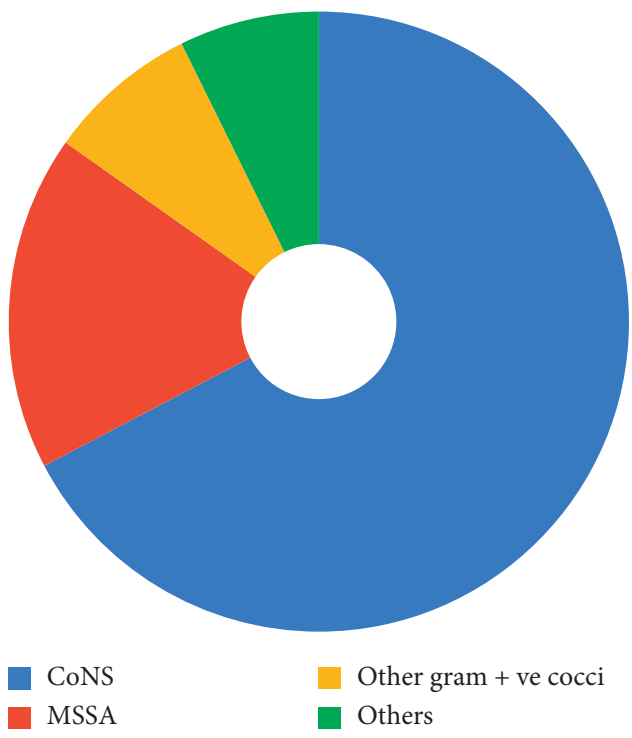

Figure 1: Distribution of bacterial isolates from HCW MPs.

$S=78.6 \%$, and penicillin and erythromycin showed the same efficacy $(S=37.7 \%)$. For CoNS, cefoxitin was also the most effective antibiotic $(S=96.5 \%)$, followed by trimethoprimsulfamethoxazole $(S=75.9 \%)$, clindamycin $(S=62.1 \%)$, penicillin $(S=31.7 \%)$, and erythromycin, which was the least influential $(S=26.3 \%)$.

It is quite clear that there are reductions in all antibiotics' susceptibility, shown by Staphylococcus isolated from the HCW MPs compared to that separated from the non-HCW. Data are more precise in $S$. aureus than CoNS.

The differences between hospital $\mathrm{A}$ and $\mathrm{B}$ concerning resistance to antibiotics are shown in Table 5. Bacterial isolate resistance against clindamycin, cefoxitin, and trimethoprim-sulfamethoxazole was more in hospital A. However, resistance against penicillin and erythromycin was more in hospital B.

MP contamination differs in distribution according to a hospital position as shown in Table 6; the highest percentage of contamination was among the residents (100\%), and the lowest was among the technicians (70\%).

\section{Discussion}

Many published articles highlighted the role of MPs as a pathway of microbial transmission. The latest systematic analysis of MP microbial contamination reveals a high overall prevalence of contamination from healthcare and community environments, hitting 68 percent [7].

More than eighty percent of HCW MPs showed microbial growth in the current study. Several other studies documented different contamination levels. Other studies performed in LMICs have demonstrated vast contamination levels $[19,20]$. Higher findings of bacterial contamination were also reported in Taiwan (94.3\%) [2], Ethiopia (94.2\%) [15], United Arab Emirates (98\%) [21], and Turkey (97.8\%) [10]. Also, our findings indicate that bacteria contaminate $86 \%$ of non-HCW MPs. This contamination level is close to research by Neha et al. [22].

Factors associated with contamination of MPs were MP owner's gender. Male HCW MPs were more contaminated, where male to female ratio was $1.5: 1$. This result is similar to a study conducted in Ethiopia [15] and Iran [23]. HCW MPusing habits showed essential associations with the contamination of MPs. Using MPs in the bathroom was associated with contamination by Gram-positive bacilli ( $p$ value $=0.026$ ).

Other data findings from the questionnaire on the MP usage behaviors, such as frequency of handwashing per day, frequency of cleaning of MPs per month, last time cleaning of MPs, cleaning method of MPs, smoking status, time spent using mobile phones per day, and hand washing after use of MPs, were checked for any correlation with laboratory results. None of these behaviors were correlated with any association.

The investigation of bacteria isolated from HCW MPs revealed that CoNS were the majority of the bacteria. The CoNS colonization average was up to $67.3 \%$ in these 
TABLE 3: Comparison of bacterial isolates between hospitals A and B.

\begin{tabular}{|c|c|c|c|c|}
\hline & \multicolumn{2}{|c|}{ Rafidia Hospital (hospital A) } & \multicolumn{2}{|c|}{ NNUH (hospital B) } \\
\hline & Percentage (\%) & Number & Percentage (\%) & Number \\
\hline Number of samples & & 100 & & 100 \\
\hline Total number of isolates & - & 212 & - & 223 \\
\hline Contamination & 87 & - & 88 & - \\
\hline \multicolumn{5}{|l|}{ Distribution of bacterial isolates } \\
\hline Gram-positive cocci: & 92.9 & 197 & 95.5 & 213 \\
\hline Staphylococci species & 83.0 & 176 & 89.7 & 200 \\
\hline CoNS & 64.2 & 136 & 70.4 & 157 \\
\hline S. aureus & 18.9 & 40 & 19.3 & 43 \\
\hline MSSA & 17 & 36 & 17.9 & 40 \\
\hline MRSA & 1.9 & 4 & 1.3 & 3 \\
\hline Other gram-positive cocci & 9.9 & 21 & 5.8 & 13 \\
\hline Gram-positive bacilli: & 5.2 & 11 & 3.1 & 7 \\
\hline Non-spore forming & 3.8 & 8 & 2.2 & 5 \\
\hline Spore forming & 1.4 & 3 & 0.9 & 2 \\
\hline Gram-negative species: & 1.9 & 4 & 1.3 & 3 \\
\hline Non-glucose fermenter & 1.4 & 3 & 0.4 & 1 \\
\hline Glucose fermenter & 0.5 & 1 & 0.9 & 2 \\
\hline
\end{tabular}

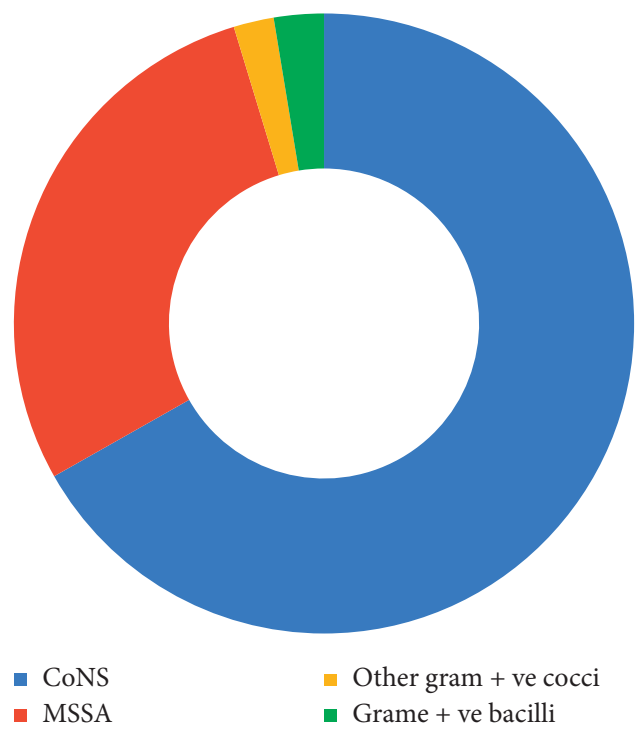

FIGURE 2: Distribution of bacterial isolates from non-HCW mobile phones.

TABLe 4: Antibiotic susceptibility pattern of Staphylococci isolated from HCW and non-HCW MPs.

\begin{tabular}{|c|c|c|c|c|}
\hline \multirow[b]{2}{*}{ Antibiotics } & \multicolumn{2}{|c|}{ Staphylococcus aureus } & \multicolumn{2}{|c|}{ Coagulase-negative Staphylococcus } \\
\hline & $\begin{array}{c}\text { HCWs } \\
\text { Susceptible (\%) }\end{array}$ & $\begin{array}{c}\text { Non-HCWs } \\
\text { Susceptible (\%) }\end{array}$ & $\begin{array}{c}\text { HCWs } \\
\text { Susceptible (\%) }\end{array}$ & $\begin{array}{c}\text { Non-HCWs } \\
\text { Susceptible (\%) }\end{array}$ \\
\hline Penicillin & 14.9 & 35.7 & 19.5 & 31.7 \\
\hline Clindamycin & 67.2 & 78.6 & 64.9 & 62.1 \\
\hline Cefoxitin & 82.1 & 100 & 91.3 & 96.5 \\
\hline Erythromycin & 23.9 & 35.7 & 23.5 & 26.3 \\
\hline Trimethoprim-sulfamethoxazole & 64.2 & 85.7 & 72.5 & 75.9 \\
\hline
\end{tabular}

samples. This outcome is consistent with other studies, such as Kokate et al. [24], Pal et al. [3], and Bodena et al. [15]. CoNS have relatively low virulence and tend to be the skin's natural flora. However, these bacteria have become increasingly recognized as the most common cause of nosocomial bacteremia associated with indwelling devices [14].
In this study, CoNS isolate percentage was lower than reported in Taiwan (90.2\%) [2] and in India (72\%) [24]. However, these isolates' percentage was higher than Egypt's results, which showed 50\% [14].

The second most common bacterial isolate from HCW MPs was S. aureus. This percentage was $17.5 \%$ MSSA and 
TABLE 5: Antibiotic resistance of hospital A and B bacterial isolates.

\begin{tabular}{lcc}
\hline Antibiotic resistance & Rafidia Hospital (hospital A) (\%) & NNUH (hospital B) (\%) \\
\hline Penicillin & 54.2 & 64.5 \\
Clindamycin & 22.6 & 15.5 \\
Cefoxitin & 5.1 & 2.50 \\
Erythromycin & 52.8 & 59.0 \\
Trimethoprim-sulfamethoxazole & 15.5 & 13.0 \\
\hline
\end{tabular}

TABLE 6: Contamination distribution according to the position in the hospital.

\begin{tabular}{lc}
\hline Position & MP contamination (\%) \\
\hline Specialists & 90.5 \\
Residents & 100.0 \\
Interns and med. students & 87.5 \\
Nurses & 87.4 \\
Technicians & 70.0 \\
Others & 83.3 \\
\hline
\end{tabular}

1.6\% MRSA. Both MSSA and MRSA are potential clinical pathogens. S. aureus can cause various illnesses, from minor skin infections to much more severe diseases. MRSA is of particular importance in the medical community, as it has evolved resistance to $\beta$-lactam antibiotics [25]. MRSA was detected on HCW stethoscopes and MPs' [26]. Tambe and Pai study in India [27] and in a community study in Italy [28] both reported that the isolation of $S$. aureus was the highest in all the categories of HCWs, $54 \%$ and $64.1 \%$, respectively. The genus of Staphylococcus was the most commonly observed isolates. It is primarily due to their ability to tolerate dryness and thus thrive and multiply rapidly in warm environments such as MPs [28].

For the other Gram-positive cocci, we found $7.9 \%$. Our findings were similar to those of Egypt [14], whereas Tambe and Pai found a higher percentage of other Gram-positive cocci in Turkey [27]. As for Gram-positive bacilli (4.2\% of total isolates), $1.2 \%$ were spore forming, and $3 \%$ were nonspore forming. In comparison, the Gram-negative bacilli were $1.6 \%$ of the total isolates, and this is considerably lower than other Ethiopia studies, where Klebsiella has been identified as the third most prevalent pathogenic bacteria [15].

As for the non-HCW group, CoNS were also the most prevalent (66.8\% of total isolates), similar to a study by Bhoonderowa et al. [28]. CoNS were also the most prevalent (69\%) bacteria from MPs of non-HCWs in Misgana et al., which reported a prevalence of 56\% [29]. As for the second most common non-HCW isolated bacteria, we found MSSA to be $28.5 \%$. Non-HCW outcomes were more outstanding than the HCW MPs, with no MRSA observed on their MPs. For the third most common isolate, the percentage of Grampositive non-spore-forming bacilli was $2.6 \%$. Additionally, $2.1 \%$ of total isolates comprised other Gram-positive cocci. No Gram-negative bacilli or Gram-positive spore-forming bacilli were isolated.

Antimicrobial resistance is the most severe health threat in treating patients (WHO). Our results showed that CoNS isolated from HCW MPs were more susceptible to cefoxitin (S=91.3\%). For trimethoprim-sulfame thoxazole, the percentage was $S=72.5 \%$, clindamycin showed $S=64.9 \%$, erythromycin showed $S=23.5 \%$, and penicillin showed $S=19.5 \%$. Regarding cefoxitin, our results showed better susceptibility than another study conducted in Iran [30] that showed $S=52.3 \%$. In contrast, CoNS in the same study had better susceptibility to erythromycin $(S=43.1 \%)$ and clindamycin $(S=71.4 \%)$ compared to our results. Among $S$. aureus isolated from the same above study group, cefoxitin was also the most effective antibiotic $(S=82.1 \%)$, followed by clindamycin $(S=67.2 \%)$, trimethoprim-sulfamethoxazole $(S=64.2 \%)$, erythromycin $(S=23.9 \%)$, and penicillin, which was the least effective ( $S=14.9 \%$ ). In our study, the prevalence of MRSA in the HCW group was $17.9 \%$. Our results showed better $S$. aureus susceptibility to cefoxitin than the Iranian study that showed $S=52.3 \%$. In contrast, the same study had better susceptibility to erythromycin $(S=43.1 \%)$ and clindamycin $(S=73.4 \%)$ than our results. This difference in antibiotic susceptibility compared to other studies might be due to different hospital environments, bacterial strains, empirical treatment practices, easy access to antibiotics without a prescription, and prolonged use of common antibiotics [30, 31].

On the other hand, isolates from non-HCW MPs showed less resistance to the last antibiotics than the HCW group. CoNS isolated from non-HCW MPs were all susceptible to cefoxitin $(S=100.0 \%)$. Results for other antibiotics were as follows: trimethoprim-sulfamethoxazole $(S=75.9 \%)$, clindamycin $(S=62.1 \%)$, penicillin $(S=31.7 \%)$, and erythromycin, which was the least influential $(S=26.3 \%)$. Among $S$. aureus isolated from the non-HCW group, cefoxitin was also the most effective antibiotic $(S=96.4 \%)$. For others, trimethoprim-sulfamethoxazole showed $S=85.7 \%$, clindamycin showed $S=78.6 \%$, and penicillin and erythromycin showed the same efficacy $(S=37.7 \%)$. No MRSA isolates were detected in the non-HCW group compared to $17.9 \%$ of S. aureus in the HCW group.

By connecting answers to the questionnaire and antibiotic resistance profile of isolates, we found that MP-using habits of HCWs were associated with increased bacterial resistance against specific antibiotics. Cefoxitin resistance was related to the existence of screen breaks on the MPs ( $p$ value $=0.042)$, entry to operating theatre $(p$ value $=0.035)$, and increased average use of MPs per day ( $p$ value $=0.011$ ). Erythromycin resistance was also associated with entry to operating theatre $(p$ value $=0.007)$. Trimethoprim-sulfamethoxazole resistance was associated with using MPs in the bathroom ( $p$ value $=0.003)$ and being a nurse or resident in a hospital position $(p$ value $=0.036)$. 
By comparison, more significant numbers of different isolates were isolated from MPs of the HCW than that of the non-HCW. Working in a hospital environment also raised the possibility of bacterial antibiotic resistance. This finding is probably due to the hospital environment and contact with patients with different infections. This increase of antibiotic resistance found in isolates from HCW MPs indicates that MPs are very likely to increase the burden of NIs. Never the less, it was found that HCWs cleaned their MPs with alcohol more frequently than non-HCWs as shown in Table 1. Not all is positive, as among HCWs, it was found that residents used their MPs in healthcare settings the most, whether it is on the entry or inside the operating theatre or beside patients. That is why it is essential to publish mandatory guidelines on the use and cleaning of MPs in healthcare settings and to take measures to reinforce them. It is interesting to mention that to present, no guidelines on the cleaning of MPs and restrictions of their use in our healthcare setting in Palestine exist.

Cross-sectional design limitations may have an impact on this research. Report bias could be introduced, by having MP handling data reported by self-reported questionnaires. Samples were obtained in all shifts on all days of the week to minimize selection bias, and it was a random process as we chose that we meet in different hospital shifts. Fortunately, because of being busy or in a hurry, only two nonresponders have been detected by the data collection process.

\section{Conclusion}

Our study shows that more significant numbers of organisms were isolated from MPs of HCWs than those of nonHCWs. CoNS were the most frequently identified bacteria contaminating MPs. It is known to be positively associated with nosocomial bacteremia. Our findings indicate that cefoxitin is the most effective antibiotic for most bacteria isolated from contaminated MPs. Being a male or a nurse, working at an operating theatre, and being aware of MPs role in spreading microbes were associated with higher contamination percentages. Also, using MPs in bathrooms increases the risk of transmission of Gram-positive bacilli. Working in a hospital environment seems to raise the possibility of bacterial antibiotic resistance.

From what precedes and from other previous studies, it looks like HCW constitutes a significant epidemiologic hazard to the public by microbial contamination of their MPs. Regular decontamination of MPs with alcohol wipes and proper hand hygiene may decrease the risk of hospitalacquired infections caused by these devices [6, 32]. Hospitals should limit MP use in the operating theatre and encourage the use of specific fixed MPs instead of personal ones to promote the application of mobile hygiene [11]. Moreover, disposable sterile MP pouches while working beside patients would help decrease MP bacterial contamination.

Additionally, it is worth studying the contamination of other instruments and devices frequently used by HCWs, such as stethoscopes and computer keyboards, by pathogenic bacteria. This would help rule out other sources of infection and contribute to stopping the cycle of nosocomial infections and saving lives [12].

\section{Data Availability}

The data used to support the findings of this study are included within the article.

\section{Conflicts of Interest}

The authors declare that there are no conflicts of interest.

\section{Authors' Contributions}

MQ was responsible for conceptualization and study design, literature search, questionnaire development, lab work, data interpretation, reviewing, and manuscript writing. RK, KA, $\mathrm{MA}$, and YN perfomed literature search, questionnaire development, lab work, analysis, data interpretation, and manuscript writing. BM carried out data analysis and interpretation, manuscript writing, and reviewing. AM and MD participated in work and analysis at lab. All the authors have read and approved the final version of the manuscript for submission.

\section{Acknowledgments}

The authors would like to acknowledge the faculty of Medicine and Health Sciences at An-Najah National University for facilitating the accomplishment of the current study. The authors are also thankful to the Palestinian Ministry of Health, Rafidia Hospital, for taking part in this study, and to An-Najah National University Hospital. The study was funded by An-Najah National University.

\section{References}

[1] R. Plowman, N. Graves, M. A. S. Griffin et al., "The rate and cost of hospital-acquired infections occurring in patients admitted to selected specialties of a district general hospital in England and the national burden imposed," Journal of Hospital Infection, vol. 47, no. 3, pp. 198-209, 2001.

[2] C. H. Chang, S. Y. Chen, J. J. Lu, C. J. Chang, Y. Chang, and P. H. Hsieh, "Nasal colonization and bacterial contamination of mobile phones carried by medical staff in the operating room," PLoS One, vol. 12, no. 5, Article ID e0175811, 2017.

[3] S. Pal, D. Juyal, S. Adekhandi et al., "Mobile phones: reservoirs for the transmission of nosocomial pathogens," Advanced Biomedical Research, vol. 4, p. 144, 2015.

[4] R. R. W. Brady, A. Wasson, I. Stirling, C. McAllister, and N. N. Damani, "Is your phone bugged? The incidence of bacteria known to cause nosocomial infection on healthcare workers' mobile phones," Journal of Hospital Infection, vol. 62, no. 1, pp. 123-125, 2006.

[5] A. Amanah, D. R. Apriyanto, and H. Fitriani, "Isolation of surveillance pathogenic fungal microbial contaminant on mobile phone," Open Access Macedonian Journal of Medical Sciences, vol. 7, no. 20, pp. 3393-3396, 2019.

[6] L. Missri, D. Smiljkovski, G. Prigent et al., "Bacterial colonization of healthcare workers' mobile phones in the ICU and effectiveness of sanitization," Journal of Occupational and Environmental Hygiene, vol. 16, no. 2, pp. 97-100, 2019. 
[7] M. Olsen, M. Campos, A. Lohning et al., "Mobile phones represent a pathway for microbial transmission: a scoping review," Travel Medicine and Infectious Disease, vol. 35, p. 101704, 2020.

[8] T. Ibrahim, A. N. Chamseddine, and M. Baz, "Mobile phones: a forgotten source of SARS-CoV-2 transmission," American Journal of Infection Control, vol. 48, no. 8, pp. 971-972, 2020.

[9] N. A. Hikmah, T. S. Anuar, and T. S. Anuar, "Mobile phones: a possible vehicle of bacterial transmission in a higher learning institution in Malaysia," Malaysian Journal of Medical Sciences, vol. 27, no. 2, pp. 151-158, 2020.

[10] C. Ustun and M. Cihangiroglu, "Health care workers' mobile phones: a potential cause of microbial cross-contamination between hospitals and community," Journal of Occupational and Environmental Hygiene, vol. 9, no. 9, pp. 538-542, 2012.

[11] N. Q. Qureshi, S. H. Mufarrih, S. Irfan et al., "Mobile phones in the orthopedic operating room: microbial colonization and antimicrobial resistance," World Journal of Orthopedics, vol. 11, no. 5, pp. 252-264, 2020.

[12] G. Messina, C. Quercioli, S. Burgassi, F. Nisticò, A. Lupoli, and N. Nante, "How many bacteria live on the keyboard of your computer?" American Journal of Infection Control, vol. 39, no. 7, pp. 616-618, 2011.

[13] H. A. Khan, F. K. Baig, and R. Mehboob, "Nosocomial infections: epidemiology, prevention, control and surveillance," Asian Pacific Journal of Tropical Biomedicine, vol. 7, no. 5, pp. 478-482, 2017.

[14] H. S. Selim and A. F. Abaza, "Microbial contamination of mobile phones in a health care setting in Alexandria, Egypt," GMS Hygiene and Infection Control, vol. 10, 2015.

[15] D. Bodena, Z. Teklemariam, S. Balakrishnan, and T. Tesfa, "Bacterial contamination of mobile phones of health professionals in Eastern Ethiopia: antimicrobial susceptibility and associated factors," Tropical Medicine and Health, vol. 47, p. $15,2019$.

[16] M. Waruingi, L. Underdahl, and W. Macharia, "Opportunity in delivery of health care over mobile devices in developing countries," African Journal of Food, Agriculture, Nutrition and Development, vol. 9, no. 5, p. 2, 2009.

[17] R. Khashei, H. S. Ebrahim-Saraie, M. Hadadi, M. Ghayem, and H. R. Shahraki, "The occurrence of nosocomial pathogens on cell phones of healthcare workers in an Iranian tertiary care hospital," Infectious Disorders-Drug Targets, vol. 19, no. 3, pp. 327-333, 2019.

[18] A. Sumritivanicha, K. Chintanavilas, and A. Apisarnthanarak, "Prevalence and type of microorganisms isolated from house staffs mobile phones before and after alcohol cleaning," Infection Control \& Hospital Epidemiology, vol. 32, no. 6, pp. 633-634, 2011.

[19] P. Datta, H. Rani, J. Chander, and V. Gupta, "Bacterial contamination of mobile phones of health care workers," Indian Journal of Medical Microbiology, vol. 27, no. 3, pp. 279-281, 2009.

[20] I. Sedighi, M. Y. Alikhani, S. Ramezani, M. Nazari, and A. S. M. Nejad, "Bacterial contamination of mobile phones of health care providers in a teaching hospital in Hamadan Province, Iran," Archives of Clinical Infectious Diseases, vol. 10, no. 2, 2015.

[21] M. Gashaw, D. Abtew, and Z. Addis, "Prevalence and antimicrobial susceptibility pattern of bacteria isolated from mobile phones of health care professionals working in Gondar town health centers," ISRN Public Health, vol. 2014, Article ID 205074, 6 pages, 2014 .
[22] S. Neha, S. Aruna, R. S. Parihar, P. K. Khatri, C. Arvind, and B. Archana, "Prevalence and antibiotic pattern of microbes isolated from mobile phones of health care workers and nonhealth care workers," International Journal of Current Microbiology and Applied Sciences, vol. 3, pp. 43-60, 2014.

[23] S. Jalalmanesh, M. Darvishi, M. Rahimi, and M. Akhlaghdoust, "Contamination of senior medical students' cell phones by nosocomial infections: a survey in a university-affiliated Hospital in Tehran," Shiraz E-Medical J, vol. 18, no. 4, 2017.

[24] S. B. Kokate, S. R. More, V. Gujar, S. Mundhe, and Q. S. Zahiruddin, "Microbiological flora of mobile phones of resident doctors," Journal of Biomedical Science and Engineering, vol. 5, no. 11, pp. 696-698, 2012.

[25] K. Becker, C. Heilmann, and G. Peters, "Coagulase-negative staphylococci," Clinical Microbiology Reviews, vol. 27, no. 4, pp. 870-926, 2014.

[26] P. Balapriya, J. Padmakumari, and A. Vijayalakshmi, "Screening for nosocomial pathogens in stethoscopes, sphygmomanometers and mobile phones of health care providers in a teritiary care hospital," International Journal of Current Microbiology and Applied Sciences, vol. 5, no. 10, pp. 91-98, 2016.

[27] N. N. Tambe and C. Pai, "A study of microbial flora and MRSA harboured by mobile phones of health care personnel," International Journal of Recent Trends in Science and Technology, vol. 4, pp. 14-18, 2012.

[28] A. Bhoonderowa, S. Gookool, and S. D. Biranjia-Hurdoyal, "The importance of mobile phones in the possible transmission of bacterial infections in the community," Journal of Community Health, vol. 39, no. 5, pp. 965-967, 2014.

[29] G. M. Misgana, K. Abdissa, and G. Abebe, "Bacterial contamination of mobile phones of health care workers at jimma university specialized hospital, Jimma, South west Ethiopia," International Journal of Infection Control, vol. 11, no. 1, pp. 1-8, 2014.

[30] R. R. Morubagal, S. G. Shivappa, R. P. Mahale, and S. M. Neelambike, "Study of bacterial flora associated with mobile phones of healthcare workers and non-healthcare workers," Iranian Journal of Microbiology, vol. 9, no. 3, pp. 143-151, 2017.

[31] A. M. Shahlol, H. M. Khalifallah, E. M. Shahlol, R. Dastidar, M. T. Halder, and G. Lamichhane, "Bacterial contamination of mobile phones and hands of health care workers in Sabha Medical Center Hospital, Fazzan area in southwestern of Libya," International Journal of Research in Medical Sciences, vol. 1, no. 4, pp. 1-8, 2015.

[32] (CDC) C of DC Detailed Disinfection Guidance, https://www. cdc.gov/coronavirus/2019-ncov/prevent-getting-sick/ cleaning-disinfection.html. 